\title{
Orbital evolution of short-period comets with high values of the Tisserand constant
}

\author{
N.Yu. Emel'yanenko \\ Department of Computational and Celestial Mechanics, South Ural University, 76 Pr. Lenina, \\ Chelyabinsk, Russia \\ email: emel@math.susu.ac.ru
}

\begin{abstract}
The orbital evolution of comets with high values of the Tisserand constant is studied for a time interval of 800 years. Scenarios of dynamical evolution are obtained for 85 comets. Particular features of the orbital evolution of the comets of this class are singled out. The orbits of all comets are tangent to the orbit of Jupiter and have a steadily low inclination. For $80 \%$ of comets, the evolution scenario includes a timespan in which the comets move in low-eccentricity orbits. The possibility is analyzed of a change in the Tisserand constant and of a transition of the comet to be controlled by other giant planets.
\end{abstract}

Keywords. Evolution, short-period comet, cyclic transformation.

\section{Introduction}

In Jupiter family, about 100 comets are known (Marsden \& Williams 2003) whose Tisserand constants satisfy the condition

$$
T_{s} \geqslant 2.9
$$

Nowadays, the orbits of these comets are tangent to the Jovian orbit at the aphelion or at the perihelion. In what follows, such orbits will normally be referred to as $A$-type ( $P$-type) orbits or denoted as $A$ and $P$, respectively. Kazimirchak-Polonskaya (1967), Everhart (1973), Carusi \& Valsecchi (1980, 1982) and Froeschlé \& Rickman (1981) have shown that such orbits are extremely unstable because of frequent encounters with Jupiter.

We study here for a time interval of 800 years the evolution of the comets whose orbits satisfy condition (1.1). Such comets are called the short-period comets of Jupiter family (JFC).

The dynamics of cometary orbits is investigated by numerically integrating the equations of motion. The Everhart method and his RADAU code (Everhart 1974) are used. The calculations are based on the systems of orbital elements borrowed from Marsden \& Williams' catalog (2003). Perturbations from nine planets are taken into account (the mass of Mercury being included in the solar mass). If a comet penetrates into the jovicentric sphere of radius $k=0.08 \mathrm{AU}$, perturbations due to the oblateness of Jupiter are taken into account (Emel'yanenko 2003a, 2003b).

The scenarios of the orbital evolution of comets are studied here for the time interval from $t_{1}=2300000.5$ to $t_{2}=2600000.5$. 


\section{Orbital evolution}

\subsection{Basic Objectives of the Study}

These are:

1) The search for peculiarities in the orbital evolution of the comets of this class.

2) Possibilities for changes in the Tisserand constant.

3) Possibilities for the transition of comets to being controlled by other giant planets.

4) The presence of persistent tendencies in the variation of cometary orbits in the past and in the future.

\subsection{Analysis of the Orbital Evolution of Comets}

We consider separately three scenarios of preferred variations in the orbital elements of the comets under study.

\subsubsection{Variations in the elements governing the spatial orientation of the orbit}

The performed investigation showed that, to a certain degree, all the comets experience variations in the angular orbital elements over the time interval $t_{1}-t_{2}$. Among all the orientation elements, let us pay particular attention to the inclination of the orbit of the comet. It is well known that different classes of comets have different distributions of the element $i$. I examined in this study the stability of the distribution of the cometary orbit inclination for the comets of Jupiter's family which obey condition (1.1) and have, by and large, extremely unstable orbits. To this end, I investigated possible transformations of the element $i$ in the approach region, total changes in the inclination over the approach time, and the evolutionary changes in the element $i$ for a time interval that encompasses 100-150 revolutions of the comet around the Sun.

Near the encounter with Jupiter, the inclination of a cometary orbit can vary over an extremely wide range. For the investigated comets, in the vicinity of the jovicentric distance minima, all the known cometary orbits with the corresponding value of the element $i$ can be found as osculating orbits.

However, the variation of the element $i$ in the encounter region is nearly symmetric with respect to the moment of the achievement of the minimum. Therefore, comets enter the encounter region and leave it at almost the same orbital inclination.

In studying evolutionary changes of the element $i$, I compared the values of the cometary orbit inclination at the moments $t_{1}$ (in the past) and $t_{2}$ (in the future).

The comets under study have a steadily low orbit inclination for the investigated time interval. In the past and future, the maximum inclination has that of the orbit of 83P /Russell $1\left(i_{1}=27^{\circ}, i_{2}=23^{\circ}\right)$.

On the whole, the performed investigation of changes in the cometary orbit inclination shows that the distribution of the element $i$ is stable over the time interval $t_{1}-t_{2}$ for the group of comets of the Jupiter family which satisfy condition (1.1).

Because of the small inclination of the cometary orbit to the ecliptic plane, changes in the two other elements specifying the spatial orientation of the orbital plane virtually do not affect the conditions of subsequent encounters of every comet with Jupiter. The proximity of the aphelion and/or the perihelion of the cometary orbit to the mean radius of the Jovian orbit is the determining factor for the occurrence of these encounters throughout the time interval at hand. 
2.2.2. Variations in the shape of a cometary orbit

We shall refer to a cometary orbit as a low-eccentricity orbit, or $l$ orbit, if its eccentricity $e$ obeys the condition

$$
e \leqslant 0.33
$$

as a medium-eccentricity orbit, or $m$ orbit, if

$$
0.33<e \leqslant 0.66
$$

and as a high-eccentricity orbit, or $h$ orbit, if

$$
e>0.66
$$

Thus, a cometary orbit will hereinafter be denoted by a letter, $A$ or $P$, with a subscript indicating the orbit eccentricity. For example, the notation $A_{m}$ corresponds to a comet whose orbit is tangent to the Jovian orbit at the aphelion and has an eccentricity satisfying condition (2.2).

Any change in the shape of a cometary's orbit and in the type of its tangency to the Jovian orbit, which occurs in the course of evolution, will be denoted by an arrow indicating the direction of the change (for example, $A_{l} \rightarrow P_{m}$ ). The evolutionary changes of the cometary orbits studied here are indicated in the first column of Table 1, with the second column giving the number of comets undergoing each evolution.

The investigation of changes in the cometary orbit shapes was aimed at resolving the following questions:

- what were the predominant eccentricities of the comets of Jupiter's family under condition (1.1) for the time interval $t_{1}-t_{2}$,

- how did the eccentricity of a cometary's orbit change near the encounter with Jupiter,

- and what particular features took place in the behavior of the eccentricity in the course of evolutionary transformations of the orbit?

The analysis of the evolution of cometary orbits showed the following: the orbits of all comets satisfy condition (2.1) or (2.2) over the studied time interval. 16 comets are constantly in medium-eccentricity $m$ orbits and three comets, in low-eccentricity $P_{l}$ or orbits $A_{l} .7$ comets are at all times in low-eccentricity orbits but undergo transitions $A_{l} \leftrightarrow P_{l}$. The other 59 comets change the shape of their orbits. All four possible scenarios of shape transformation take place: either the form of a cometary's orbit changes without the reversal of tangency (transitions $A_{m} \leftrightarrow A_{l}, P_{m} \leftrightarrow P_{l}$ ), or both the form and the type of tangency to the Jovian orbit are changed (transitions $P_{m} \leftrightarrow P_{l}, P_{m} \leftrightarrow A_{l}$ ).

It is the encounters with Jupiter that directly affect the changes of the element $e$. Deeply in the sphere of action of Jupiter, the osculating eccentricity values vary over an extremely wide range, up to the hyperbolic value. However, as consequence of the encounter, only two scenarios take place in the behavior of the eccentricity function: the element $e$ either changes (according to the scheme $m \rightarrow l$ or $l \rightarrow m$ ) or remains unchanged (the orbit of the comet satisfying condition (2.1) or (2.2) both before and after the encounter).

For the comets studied, the main feature of the orbit-eccentricity behavior is the presence of a timespan $\Delta t$ during which these comets move in $P_{l}$ or $A_{l}$ orbits. For 68 comets $(80 \%)$, the evolution scenario includes at least one such timespan $\Delta t$ in the interval $t_{1}-t_{2}$.

\subsubsection{Changes in the sizes of cometary orbits}

In considering changes in the cometary's orbit sizes, the principal attention was given to the following questions: what are the orbit sizes of the comets under study in the time interval $t_{1}-t_{2}$ what particular features are related to the orbit sizes of the comets 
Table 1. Short-period comets of Jupiter's family with high values of the Tisserand constant. Evolutionary transitions

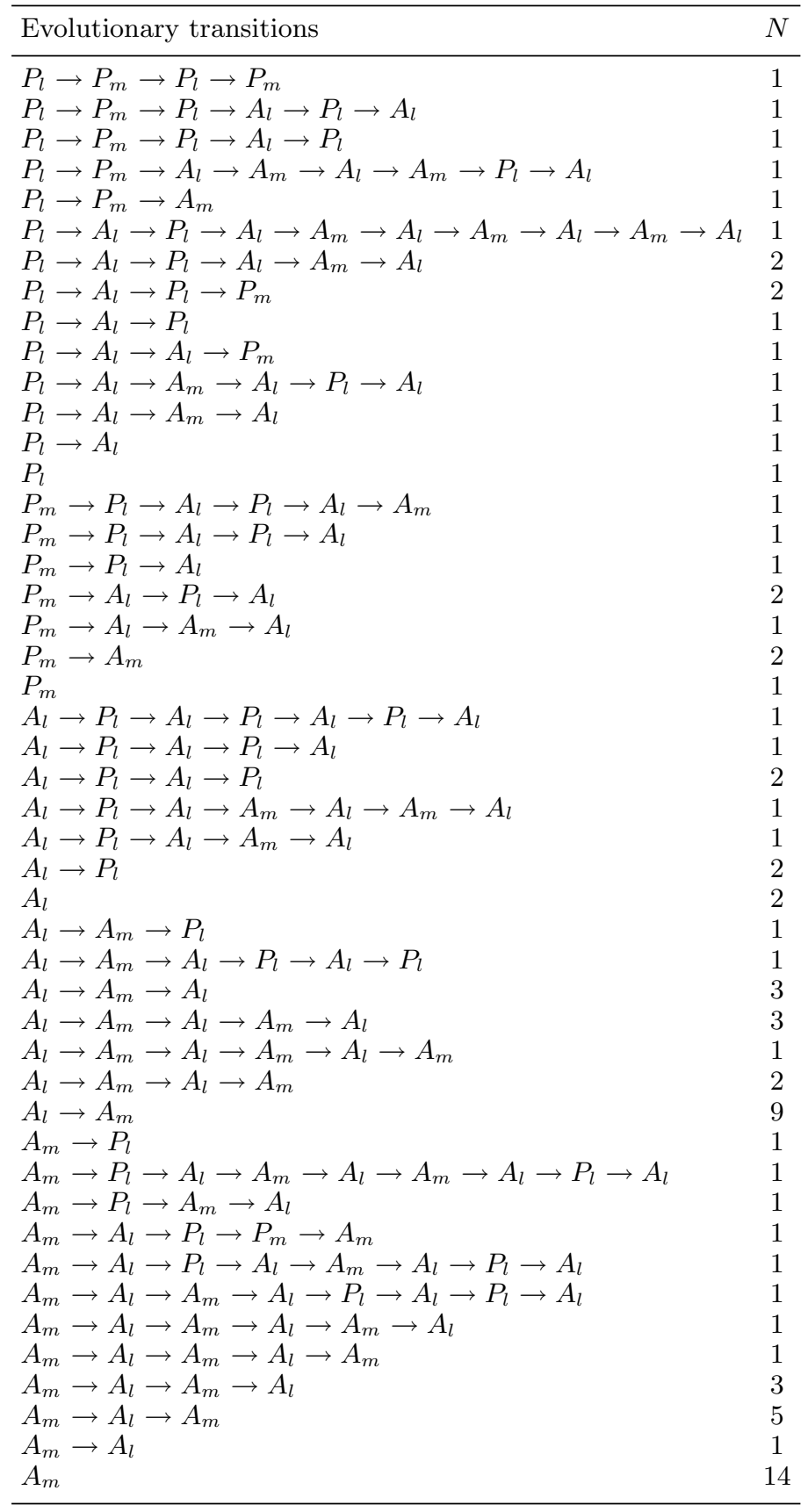

of a given class, and what are the transformations in the orbit size which occur during encounters with Jupiter?

116P/Wild 4 had the minimum orbit size $\left(a_{\min }=2.90 \mathrm{AU}\right)$ and P/1997 T3 LagerkvistCarsenty the maximum size $\left(a_{\max }=12.03 \mathrm{AU}\right)$. 
The basic feature related to the sizes of the cometary orbits is their permanent tangency to the orbit of Jupiter over the 800-yr time interval. For the orbits tangent at the perihelion, $q_{\text {min }}=3.71 \mathrm{AU}(\mathrm{P} / 1997 \mathrm{~V} 1$ Larsen$)$ and $q_{\max }=6.74 \mathrm{AU}$ (P/1999 XN120 Catalina). For the orbits tangent at the aphelion, $Q_{\text {min }}=4.63 \mathrm{AU}$ P/2001 YX127 LINEAR and $Q_{\max }=6.06 \mathrm{AU}(82 \mathrm{P} /$ Gehrels 3).

An estimate of the lifetime of comets in $A$ and $P$ orbits indicates the following. There are 44 comets that move in an $A$ orbit over the entire time interval considered and 2 comets that are constantly in a $P$ orbit (see Table 1 ). The minimum lifetime is equal to three revolution periods for both $A$-type (39P/Oterma) and $P$-type (74P/SmirnovaChernykh) orbits.

We shall use the above-proposed criteria and notation in analyzing the transformations of orbits which take place at encounters with Jupiter. As it was noted in the discussion of changes in the shape of a cometary orbit, the comets satisfying condition (1.1) have $m$ or $l$ orbits. Therefore, the comets under study can have only $A_{m}, A_{l}, P_{m}$ and $P_{l}$ orbits.

Changes in the orbit sizes of these comets occur in two ways: either the type of tangency between the orbits of the comet and Jupiter remains invariant, and a close encounter transforms the aphelion distance of $P$-type comets or the perihelion distance of $A$-type comets (transitions $P_{m} \leftrightarrow P_{l}, A_{m} \leftrightarrow A_{l}$ ), or reversal of tangency takes place, and a close encounter with Jupiter transforms both the aphelion and the perihelion distance of the comet (transitions $P_{m} \leftrightarrow A_{m}, P_{l} \leftrightarrow A_{l}, P_{m} \leftrightarrow A_{l}, P_{l} \leftrightarrow A_{m}$ ).

The same comet can undergo both first-type and second-type transitions (see the first column of Table 1).

Even a very close encounter with Jupiter can leave the sizes of the cometary orbit virtually unchanged (for the approach of $147 \mathrm{P} /$ Kushida-Muramatsu, $T_{p}=2391334 \mathrm{JD}$, $\left.\rho=0.00107 \mathrm{AU}, \Delta 1 / a=-0.00022 \mathrm{AU}^{-1}\right)$.

Let us examine the past and the future of the comets, using Table 1.

Among the comets studied, the number of objects with $A$ orbits shows a decrease in the future and, even more significant, in the past. According to the Table, the nearest past and the future of a certain amount of comets with $A$ orbits demonstrate a redistribution of orbits: these are the objects whose orbits are tangent to the Jovian orbit at the perihelion. They exhibit a great variance in aphelion distances: $Q_{\max }=19.06 \mathrm{AU}(\mathrm{P} / 1997 \mathrm{~T} 3$ Lagerkvist-Carsenty), whereas $Q_{\min }=4.63 \mathrm{AU}(\mathrm{P} / 2001$ YX127 LINEAR).

No concentration of aphelia in the neighborhood of the orbits of Saturn or Uranus is observed.

The exhaustion of $A$ orbits revealed for arbitrarily chosen moments $t_{1}$ and $t_{2}$ evidences that comets obeying condition (1.1) spend a finite time in short-period orbits.

The current presence of $A$-type orbits supports the fact that this group is constantly replenished at the expense of comets with $P$ orbits.

The redistribution of orbits in the past and in the future is the result of transitions between $A$ and $P$ type orbits common for the investigated comets: 36 comets $(47 \%)$ have a total of 85 transitions $A \leftrightarrow P ; 21$ comets undergo cascade transitions $P \rightarrow A \rightarrow P ; 21$ demonstrate cascade transitions $A \rightarrow P \rightarrow A ; 10$ comets show only $P \rightarrow A$ transitions; and 4 comets, only $A \leftrightarrow P$ transitions. It can be suggested that any comet presented in Table 1 has cascade transitions $P \rightarrow A \rightarrow P \rightarrow A \rightarrow \ldots$ but the encounters with Jupiter responsible for these transitions are beyond the investigated time interval.

Now we summarize some results of the analysis of the orbital evolution of comets.

The comets under consideration have orbits with a steadily low inclination, which are tangent to the Jovian orbit at the aphelion or at the perihelion. The eccentricity of their orbits does not exceed $e=0.6$. Of these comets, $80 \%$ have a timespan $\Delta t$ in their evolution during which they move in low-eccentricity orbits. Residence in an $l$ orbit 
increases the instability of the orbit itself and, evidently, of the comet moving in it as well. The instability is due to very efficient encounters with Jupiter which took place during the timespan. Such encounters can be accompanied not only by transitions $A \leftrightarrow P$ but also by multiple minima of the jovicentric-distance function of the comet and by its temporary satellite capture (TSC).

At the same time, only cascade transitions $A \rightarrow P \rightarrow A \rightarrow \ldots$ take place for the comets studied, i.e., their orbits evolve during at least 100-150 revolutions about the Sun on a closed cycle limited by two types of tangency to the Jovian orbit. No hyperbolic ejection of a comet is revealed. As shown by Kresák (1977), hyperbolic ejections are impossible for comets with high values of the Tisserand constant.

\subsection{Cyclic Transformations of Cometary Orbits}

Now we shall examine cyclic transformations of the orbits of comets in greater detail.

For the investigated comets, the most substantial of the known orbit-size transformations follow scheme I:

$$
P_{m} \leftrightarrow A_{m} .
$$

Such transitions also take place for comets that do not satisfy condition (1.1). They occur in very close approaches of D/1770 L1 Lexell, 76P/West-Kohoutek-Ikemura and 81P/Wild 2. Such encounters are rare, while, as shown by Everhart (1973), the efficiency of more frequent but less close encounters is on the whole small.

The known transitions $A \leftrightarrow P$ of comets which do not satisfy condition (1.1) do not place the comet in a low-eccentricity orbit. Encounters with Jupiter which result in transitions $A \leftrightarrow P$ either leave the comet in a medium-eccentricity $m$ orbit (in a higheccentricity $h$ orbit) or cause changes in the shape of the cometary orbit according to the scheme $m \leftrightarrow h$. In such encounters, the Öpik model for the change of the heliocentricvelocity vector of the comet is realized. Such encounters result in transitions $A \leftrightarrow P$, if the change corresponds to a transformation of this type. Since very close encounters with Jupiter are rare in occurrence, none of the comets not satisfying condition (1.1) has a cascade $P \rightarrow A \rightarrow P$ within the time interval $t_{1}-t_{2}$. This interval is too short for investigating the orbital evolution of such comets.

For comets satisfying condition (1.1), four close approaches with transitions $P_{m} \rightarrow A_{m}$ that follow scheme I (2.4) are known. However, as the analysis of the orbital evolutions shows, the great majority of comets pass in all transitions $A \leftrightarrow P$ either from a loweccentricity $l$ orbit or to a $l$ orbit; in most cases of the encounters with Jupiter which lead to transitions $A \leftrightarrow P$, the shape of the cometary's orbit changes insignificantly (so that the comet has an $l$ orbit both before and after the encounter).

Hence, for the comets studied, evolutionary transformations $P_{m} \leftrightarrow A_{m}$ can be realized by stages, according to the scheme II:

Scheme II (Fig. 1) includes an intervening stage, the transition of the comet to loweccentricity $P_{l}$ or $A_{l}$ orbits.

While a transition by scheme I (2.4) requires a very close approach to Jupiter, each of the transitions of scheme II (Fig. 1) is realized as early as at entering the Jovian sphere of action; moreover, the penetration of the comet into the sphere of unit radius (1 AU) centered at this planet is in many cases sufficient for transitions $P_{l} \leftrightarrow A_{l}$.

Thus, the type of tangency of the orbits of Jupiter and a comet that satisfies condition (1.1) can change not only according to scheme I (2.4), but also according to scheme II (Fig. 1). The motion of real comets according to the scheme II (Fig. 1) leads to a fairly fast orbital evolution of the comets of this class even over a 800-yr time interval. This makes it possible to reveal and investigate the basic laws of evolutionary transformations 




Figure 1. Scheme II.

of cometary orbits considering a time interval shorter than in the case of comets with lower values of the Tisserand constant.

For the comets studied, all the particular transitions of scheme II (Fig. 1) take place. For all the comets, in general, most transitions are realized many times. There are comets that repeatedly undergo the transitions of the central and the right portion of the scheme. Four comets have passed from a $P_{m}$ to an $A_{m}$ orbit by scheme II (Fig. 1), choosing one or another sequence of transitions. Note that there are two types of transitions in the scheme which involve only changes in the shape of the cometary orbit (transitions $P_{m} \leftrightarrow P_{l}$ and $\left.A_{m} \leftrightarrow A_{l}\right)$, as well as the above-discussed transitions $P_{l} \leftrightarrow A_{l}$ involving only changes in the orbit sizes. We see that these transitions form an ingredient of scheme II (Fig. 1) and actually take place for real comets (see Table 1).

Scheme II (Fig. 1) includes three basic states of a cometary orbit:

- State $A_{m}$. This is a state of relative stability, which is enhanced if the orbit lies within the Jovian orbit. There are 66 comets that passed through state $A_{m} .14$ comets remain in $A_{m}$ orbits for the whole time interval studied.

- State $P_{l}$ or $A_{l}$. These states were passed by 67 comets. Three comets have $P_{l}$ or $A_{l}$ orbits over the entire time interval $t_{1}-t_{2}$. Seven comets undergo only transitions $P_{l} \leftrightarrow A_{l}$. The transitions of the central and the right portion of the scheme leave the Tisserand constant virtually unchanged. There are comets that evolve within these two parts of the scheme, undergoing up to seven transitions over the entire time interval at hand.

- State $P_{m}$. Only 14 comets passed through state $P_{m}$. In the transitions of the left portion of the scheme, the largest change in the Tisserand constant is observed. For the comets listed in Table 1, this is an unstable state. None of them have spent the whole studied time interval in this orbit. The instability is determined not only by Jupiter but also by the influence of other great planets: eight comets have encounters with Saturn and one, with Uranus. At the same time, none of the comets, being in an $P_{m}$ orbit, broke free of Jupiter's control. Thus, the comets that satisfy condition (1.1) evolve according to scheme II (Fig. 1) for a long time interval that encompasses their 100-150 revolutions about the Sun. Their orbital ellipses pulsate in their sizes, shape, and the type of tangency with the Jovian orbit. The giant planet holds these comets steadily and tightly in its possessions in spite of their "walks" over scheme II (Fig. 1) and "dizzy" jumps according to scheme I (2.4), which is also admissible for them. 


\section{Discussion}

We proposed evolutionary scheme II (Fig. 1) for comets of the Jupiter family with high values of the Tisserand constant. The transformations of the orbits of all the studied comets follows this scheme.

It is beyond doubt that these objects are the most rapidly evolving comets in the Solar System: 85 transitions $A \leftrightarrow P$ and more than 130 transitions $m \leftrightarrow l$ were revealed for them.

In the evolution scenarios of 68 comets (80\%), a timespan $\Delta t$ is present over which the comet moves in a low-eccentricity $l$ orbit, and 12 comets are in $l$ orbits for the 800 yr interval. The presence of this timespan plays an important role in the evolutionary transformations of cometary orbits subject to condition (1.1) and is the main cause of the instability of these orbits.

Low-velocity approaches of these comets to Jupiter are in many cases accompanied by the temporary satellite capture (TSC) of the comet and multiple minima of its jovicentricdistance function. Such encounters result from a similarity between the $l$ orbits and the Jovian orbit for the timespan $\Delta t$.

The following evolutionary scenarios are known exclusively for comets with $l$ orbits: comet $\leftrightarrow$ temporary satellite of Jupiter; comet $\rightarrow$ temporary satellite of Jupiter $\rightarrow$ comet disruption $\rightarrow$ many comets. The last factor possibly evidences that $l$ orbits are the primordial orbits of the comets of Jupiter's family which obey condition (1.1). The following arguments in favor of this suggestion can be noted. First, the Tisserand constant of the investigated comets satisfies condition (1.1) for the 800-yr time interval (and for $l$ objects, it satisfies this condition). Second, in the evolution scenarios of $80 \%$ of comets, the timespan $\Delta t$ is present. Third, the above-proposed scheme can be realized (i.e., the entire multitude of the orbits of these comets can be obtained from $l$ orbits). We do not necessarily imply that such comets form immediately in the capture zone. One cannot rule out the possibility of another factor that transforms some comets or other objects into comets of this class. However, what follows is their long-term evolution according to the proposed scheme.

\section{Acknowledgements}

This work was supported by RFBR Grant 06-02-16512. The author would like to thank Giovanni Valsecchi for helpful comments.

\section{References}

Carusi, A. \& Valsecchi, G.B. 1980, Moon \& Planets 22, 113

Carusi, A. \& Valsecchi, G.B. 1982, in: G. Teleki \& W. Fricke (eds.), Sun and Planetary System, (Dordrecht: D. Reidel) p. 379

Emel'yanenko, N.Yu. 2003a, Solar System Research 37/1, 66

Emel'yanenko, N.Yu. 2003b, Solar System Research 37/2, 156

Everhart, E. 1973, Astron. J. 78, 329

Everhart, E. 1974, Celest. Mech. 10, 35

Froeschlé, Cl. \& Rickman, H. 1981, Icarus 46, 400

Kazimirchak-Polonskaya, E.I. 1967, Astron. Zh. 44, 439

Kresák, L. 1977, in: A.H. Delsemme (ed.) Comets, Asteroids, Meteorites Interrelations, Evolution and Origins (Univ. of Toledo), p. 313

Marsden, B.G. \& Williams G.V. 2003, Catalogue of Cometary Orbits 